Hier steht eine Anzeige.

仿 Springer 
Paediatr Paedolog 2013 · [Suppl 1] 48:6-9

DOI 10.1007/s00608-013-0081-7

๑) Springer-Verlag Wien 2013

\author{
F. Waldhauser ${ }^{1} \cdot$ R. Püspök ${ }^{2} \cdot$ E. Tatzer ${ }^{3} \cdot$ L. Thun-Hohenstein ${ }^{4}$ \\ ${ }^{1}$ Wien \\ ${ }^{2}$ Halbturn \\ ${ }^{3}$ Hinterbrühl \\ ${ }^{4}$ Salzburg
}

\section{Kinder- und jugendmedizinische Primärversorgung in Österreich}

„hallo! wir mussten gestern bei unserm KIA 4 stunden lang warten. ich finde das wirklich enorm“ [1]. Mit diesem Eintrag beginnt eine Mutter im Internetforum "Parents and more“ einen neuen Diskussionsblock über überbordende Wartezeiten beim Kinderarzt. Nach 28 Einträgen ist man sich einig: Man wechselt den Kassenarzt oder sucht besser einen Wahlarzt.

Demgegenüber klagen die in Wien niedergelassenen Kinderärzte mit allen Kassen, dass sie im Schnitt täglich 50 bis mehr als 67 Kinder zu versorgen haben. Die Notfallambulanzen der städtischen Kinderspitäler stöhnen über eine alle Grenzen sprengende Zunahme der Ambulanzfrequenzen im letzten Jahrzehnt. All das, obwohl Wien die höchste Dichte an Kassenärzten in Österreich hat. In der „Provinz“, besonders im ländlichen Raum, wird die Primärversorgung überwiegend von den Allgemeinmedizinern und nicht von den Kinderärzten getragen - so die Statistiken der Österreichischen Krankenversicherungsträger.

In Lancet wird vorgerechnet, dass Österreich im Vergleich zu Schweden in den Jahren 2006-2010 jährlich 106 "excess deaths" unter den Kindern bis 14 Jahren hatte [2], die UNICEF $[3,4]$ und die OECD [5] stellen Österreich ein denkbar schlechtes Zeugnis für seinen Umgang mit der Kindergesundheit aus. Die Gesundheitsökonomen rechnen vor, dass wir mit den Ausgaben im Mittelfeld der industrialisierten Länder liegen, die Effizienz der investierten Mittel aber zu wünschen übrig lässt. Die Kinderärzte sind unter den am schlechtesten verdienenden Fachärzten Österreichs. Nur die österreichischen Kinder und Eltern sind mehrheitlich mit dem System recht zufrieden.

\section{\) Die Effizienz der investierten Mittel lässt zu wünschen übrig}

Haben wir nun ein Problem in der gesundheitlichen Primärversorgung der Kinder in Österreich? Haben die Österreicher ein Wahrnehmungsdefizit?

Diesen und ähnlichen Fragen ist die Politische Kindermedizin (PKM) auf ihrer letzten Jahrestagung in Klagenfurt nachgegangen. Die Referenten dieser Tagung konnten überzeugt werden, den Inhalt ihrer Präsentationen auch in schriftlicher Form abzufassen, weitere Experten wurden gewonnen, um die Fragen zu vertiefen. Das Ergebnis ist eine Momentaufnahme der Primärversorgung der Kinder und Jugendlichen in Österreich mit einem vergleichenden Blick in das europäische Ausland.

J. Ehrich, emeritierter Leiter der Pädiatrie II in Hannover, und langjähriger Sekretär und Präsident der European Paediatric Association (EPA/UNEPSA), arbeitet seit Jahren an pädiatrischen Versorgungsfragen auf europäischer Ebene. Er stellt die durchaus unterschiedlichen Primärversorgungsmodelle für Kinder in Europa vor und lässt seine Präferenz für ein integriertes Model nach schwedischem Vorbild nicht missen. E. JägerRoman, niedergelassene Kinderärztin in Berlin und Präsidentin der European Confederation of Primary Care Paediatricians, spricht die europaweite Diskussion über die Vor- und Nachteile von Allgemeinmedizinern bzw. von Kinderärzten als Primärversorger an. Unbeeinflusst von dieser Diskussion sieht sie die Zukunft der Kinderversorgung in einer besseren Qualifikation der jeweiligen Versorger.
Die einstige diplomierte Kinderkrankenschwester und jetzt weit über Österreich hinaus bekannte Gesundheitsökonomin M. Hofmarcher belegt beeindruckend, dass die Kinderversorgung in Österreich mit den Kosten im Mittelfeld liegt, aber leider auch im Effekt nur Mittelmaß ist. Einen wertvollen Schatz an Zahlen, Daten und Fakten zum österreichischen Gesundheitsversorgungsnetz für Kinder und Jugendliche liefern $P$. Winkler und der jetzige Leiter der Gesundheit Österreich GmbH (GÖG), G. Ziniel; sie haben aber auch klare Empfehlungen für die Prävention und integrierte Versorgung der „modernen Morbiditäten“.

M. Prunbauer, Fachbereichsleiter in der NÖ Patienten- und Pflegeanwaltschaft, zeigt die Arbeitsweise der Patientenanwaltschaft auf und berichtet, dass erfreulicherweise wenige Fälle aus der Kindermedizin an sie herangetragen werden. L. Damm, Allgemeinmedizinerin, Expertin in der Gesundheitsverwaltung und jetzt am Institut für Umwelthygiene der Medizinischen Universität Wien für Child Public Health verantwortlich, analysiert aus ihrem Blickwinkel die Realität der Primärversorgung unserer Kinder und Jugendlichen.

Die bisher genannten Beiträge kommentieren die Primärversorgung von außen - durchaus auch im Vergleich mit dem europäischen Ausland. Die Innenansicht durch die Betreuer eröffnet ein Artikel von R. Glehr, dem Präsidenten der Österreichischen Gesellschaft für Allgemein- und Familienmedizin, über deren Möglichkeiten und Grenzen in der Versorgung von Kindern und Jugendlichen. Der Darstellung kommt besondere Bedeutung zu, weil die Primärversorgung 
quantitativ zum größeren Teil durch Allgemeinmediziner getragen wird.

R. Püspök et al. geben mit der wahrscheinlich bisher größten Umfrage unter den niedergelassenen Kinderärzten Österreichs mit einer Rücklaufquote von mehr als 70\% eine repräsentative Innenansicht der Primärversorgung durch die Pädiater wider. Aufgrund ihres Umfangs und Detailreichtums wird die Umfrage in einem eigenen Abschnitt präsentiert. Ergänzt wird das Bild durch die Darstellung der Aktivitäten der Notfallambulanzen an den Kinderspitälern in den großstädtischen Ballungsräumen (D. Koller, langjähriger Leiter der Allgemeinen Ambulanz an der Kinderklinik im AKH Wien, u. L. Damm) und im ländlichen Raum (R. Kerbl, Primarius an der Abteilung für Kinder und Jugendliche, LKH Leoben).

Zweifelsohne wird die pädiatrische Primärversorgung in Österreich ganz überwiegend durch in Einzelpraxen tätige Kinderärzte wahrgenommen. Vereinzelt haben sich aber auch alternative Modelle entwickelt: $P$. Voitl, Gründer und ärztlicher Leiter des größten österreichischen nichtspitalsgebundenen kinderfachärztlichen Versorgungszentrums, zeigt den Stellenwert und die Entwicklungschancen pädiatrischer Gruppenpraxen und Ambulatorien für die pädiatrische Primärversorgung auf.

G. Melzer berichtet über ihre 24-jährige Tätigkeit als mobile Kinderkrankenschwester in einer niederösterreichischen Kleinstadt in Kooperation mit einem niedergelassenen Kinderarzt. Sie praktiziert seit Langem das, was im schwedischen Versorgungsmodell der Aufgabenbereich einer „community nurse“ ist.

\section{)> Die Defizite in der}

Primärversorgung psychiatrisch auffälliger Kinder sind gravierend

Der Stellenwert der psychiatrischen Erkrankungen und Therapien rückt zunehmend in das öffentliche Bewusstsein. Dies gilt auch für die Kindermedizin und die kindermedizinische Primärversorgung. $R$. Fliedl zeigt anhand epidemiologischer Daten und seiner Erfahrung als Primarius der Abteilung für Kinder- und Jugendpsy- chiatrie und Psychotherapie Hinterbrühl, welche gravierenden Defizite in der primären Versorgung von Kindern mit psychiatrischen Auffälligkeiten bestehen. C. Hartl, Obfrau der Fachgruppe Kinderund Jugendpsychiatrie, hat als erste Fachärztin für Kinder- und Jugendpsychiatrie eine Kassenstelle in Niederösterreich erkämpft und berichtet über die Patienten, die sie in ihrer Praxis sieht und zusammen mit Therapeuten anderer Spezialitäten in einem Netzwerk ambulant betreut. Ihre gesamte Tätigkeit wird von einer laufenden Evaluation begleitet. Dem Thema der begleitenden Qualitätskontrolle ist ein abschließender Artikel von G. Spiel, dem Gründer, Geschäftsführer und ärztlichen Leiter von pro mente: kinder jugend familie $\mathrm{GmbH}$, mit Kollegen gewidmet. Der Beitrag präsentiert die Ergebnisse einer begleitenden 3-jährigen Evaluation der Tätigkeiten in den Ambulatorien von pro mente, in denen Patienten mit Entwicklungs- und/oder psychischen Störungen interdisziplinär betreut werden.

Unter Leitung von R. Kerbl, dem jetzigen Präsidenten der Österreichischen Gesellschaft für Kinder- und Jugendheilkunde, diskutiert ein hochkarätig besetztes Podium zentrale Fragen der Primärversorgung: D. Baumgartner, Obmann der Fachgruppe für Kinder- und Jugendheilkunde und Vizepräsident der Ärztekammer für Niederösterreich; C. Klein, stellvertretender Generaldirektor des Hauptverbands der österreichischen Sozialversicherungsträger; V. Wolschlager, Leiterin der Koordinationsstelle für Kinderund Jugendgesundheit im Bundesministerium für Gesundheit, und die beiden bereits vorgestellten M. Hofmarcher und E. Jäger-Roman.

Den Abschluss bildet eine Resolution, die von den Teilnehmern der 6. Jahrestagung konzipiert und von der Politischen Kindermedizin (PKM) ausformuliert wurde. Es werden zentrale Themen mit dringendem Handlungsbedarf angesprochen, die die gesundheitliche Situation der Kinder in Österreich verbessern könnten. Die Themen werden an die Öffentlichkeit und an die Politik herangetragen und bilden zentrale Arbeitsschwerpunkte der PKM für die unmittelbare Zukunft.

Zusätzliche Brisanz hat die Diskussion über die Primärversorgung durch die jüngst getroffene Einigung der politischen Interessengruppen zur Gesundheitsreform erlangt, die der Primärversorgung einen gewichtigeren Stellenwert im österreichischen Gesundheitssystem einräumt als bisher [6]. Details sollen unter Federführung der GÖG in den nächsten Monaten erarbeitet werden.

\section{IL Weletrouser}

F. Waldhauser



R. Püspök



E. Tatzer

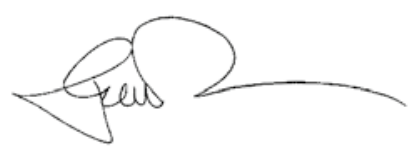

L. Thun-Hohenstein

Wien, im April 2013

\section{Korrespondenzadresse}

Prof. Dr. F. Waldhauser

Amerlingstr. 4/20, 1060 Wien

Österreich

f.waldhauser@aon.at

\section{Literatur}

1. Parents and more. http://www.parents.at/forum/ showthread.php?t=591657\#.UWKKLDfsByY. Zugegriffen: 7. April 2013

2. Wolfe I, Thompson M, Gill P et al (2013) Health services for children in western Europe. Lancet 381:1224-1234

3. http://www.unicef-irc.org/publications/pdf/rc7 eng.pdf. Zugegriffen: 10. April 2013

4. http://www.unicef-irc.org/publications/pdf/rc11_ eng.pdf. Zugegriffen: 10. April 2013

5. http://www.oecd.org/social/family/43590132.pdf. Zugegriffen: 10. April 2013

6. http://www.bmg.gv.at/cms/home/attachments/1/7/4/CH1004/CMS1363768014239/vorblatt_gesundheitsreformgesetz2013e_m.pdf 
Hier steht eine Anzeige.

黛 Springer 